In the present economic circumstances, Revue de Métallurgie intends to maintain through year 2009 its task of knowledge dissemination and technology transfer in service of the steelmaking industry.

In keeping with the previous years, Revue de Métallurgie will be published as a paper version and on the internet (www.revue-metallirgie.org).

Since October 2000, the on-line edition has built up a unique collection on the order of 800 papers with free access for subscribers and with an alternative pay per view access. The full colour on-line edition affords a search tool by authors and key words.

As from year 2009, Revue de Métallurgie will offer on-line advertisement to interested companies, as an alternative to the traditional paper advertisement.

Revue de Métallurgie will not increase its subscription rate in 2009; thus, it will have been kept stable for three consecutive years.
Taking into account the current decline of the number of potential subscribers and the global reduction of advertisement, the keeping of our publication strongly depends on the support of our readers and on the interest they take in a long term approach of knowledge diffusion.

Depending on the future economic circumstances, and in order to maintain its essential assignment, Revue de Métallurgie may have to give up the complimentary and not contractual distribution of copies of the steel research international journal. Indeed the editorial line of this journal meets the priority expectations of a minority of our subscribers only and the purchase of these issues constitutes a significant expense for our company.

Revue de Métallurgie gratefully acknowledges its subscribers for granting their renewed confidence through year 2009 and will welcome any suggestion to improve the service they are provided with.

\section{Bernard CRETON}

Chairman and Managing Director 
Dans un contexte économique globalement difficile, la Revue de Métallurgie entend poursuivre en 2009 sa mission de diffusion des connaissances et de transfert de technologies au service de l'industrie sidérurgique.

Comme les années précédentes, La Revue de Métallurgie sera publiée sous forme papier et en version électronique sur internet (www.revue-metallurgie.org).

La version mise en ligne depuis octobre 2000 constitue une base de données unique d'environ 800 articles en libre accès pour les abonnés ou accessibles par paiement en ligne.

La version en ligne dispose d'un moteur de recherche par noms d'auteurs et par mots clés et offre l'avantage d'une présentation des figures en couleurs.

Dès 2009, La Revue de Métallurgie offrira à ses annonceurs la possibilité de publier de la publicité en ligne en complément de la publicité traditionnelle en version imprimée.

La Revue de Métallurgie n'augmentera pas ses tarifs d'abonnements en 2009; ainsi ils resteront stables pour la troisième année consécutive.

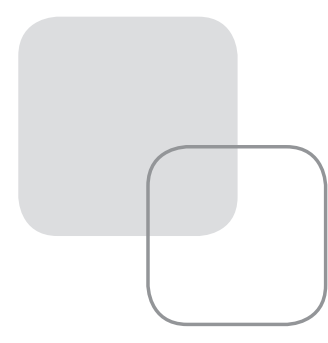

Compte tenu de l'érosion conjoncturelle du nombre d'abonnés potentiels et de la diminution générale des dépenses de publicité, le maintien de notre publication dépend tout particulièrement du soutien de ses lecteurs et de l'intérêt qu'ils veulent bien accorder à une démarche à long terme de diffusion des connaissances. En fonction de l'évolution des circonstances économiques et pour préserver l'essentiel de sa mission, La Revue de Métallurgie pourrait être amenée à renoncer à la mise à disposition gratuite et non contractuelle de la revue steel research international. En effet la ligne éditoriale de cette publication scientifique ne répond aux attentes prioritaires que d'une minorité de nos abonnés et l'achat des numéros constitue une charge financière significative pour notre société.

La Revue de Métallurgie remercie par avance ses abonnés de bien vouloir lui renouveler leur confiance en 2009 et recueillera avec plaisir toutes les suggestions d'amélioration qu'ils pourront faire pour mieux les satisfaire.

\section{Bernard CRETON}

Président Directeur Général 